\title{
Effects of $\alpha$-ketoglutarate on energy status in the intestinal mucosa of weaned piglets chronically challenged with lipopolysaccharide
}

\author{
Yongqing Hou ${ }^{1 *}$, Kang Yao ${ }^{2,3}$, Lei Wang ${ }^{1}$, Binying Ding ${ }^{1}$, Dabo Fu ${ }^{1}$, Yulan Liu ${ }^{1}$, Huiling Zhu ${ }^{1}$, Jian Liu ${ }^{1}$, \\ Yongtang $\mathrm{Li}^{1}$, Ping Kang ${ }^{1}$, Yulong Yin ${ }^{2}$ and Guoyao $\mathrm{Wu}^{3,4 *}$ \\ ${ }^{1}$ Hubei Key Laboratory of Animal Nutrition and Feed Science, Wuhan Polytechnic University, Wuhan 430023, People's \\ Republic of China \\ ${ }^{2}$ Institute of Subtropical Agriculture, The Chinese Academy of Sciences, Changsha 410125, People's Republic of China \\ ${ }^{3}$ Department of Animal Science, Texas AGM University, College Station, TX 77843, USA \\ ${ }^{4}$ State Key Laboratory of Animal Nutrition, China Agricultural University, Beijing 100193, People's Republic of China
}

(Received 20 July 2010 - Revised 7 January 2011 - Accepted 10 January 2011 - First published online 23 February 2011)

\section{Abstract}

The present study determined whether $\alpha$-ketoglutarate (AKG) might affect the expression of AMP-activated protein kinase (AMPK) and energy status in the intestinal mucosa of piglets challenged with Escherichia coli lipopolysaccharide (LPS). A total of eighteen piglets (weaned at $21 \mathrm{~d}$ of age) were allocated to one of three treatments: (1) non-challenged (control); (2) LPS-challenged (LPS); (3) LPS $+1 \%$ AKG (LPS + AKG). Piglets in the control and LPS groups were fed a maize- and soyabean meal-based diet, and the LPS + AKG group was fed the basal diet supplemented with 1\% AKG. On days 10, 12, 14 and 16 of the trial, piglets in the LPS and LPS + AKG groups were challenged with LPS $(80 \mu \mathrm{g} / \mathrm{kg}$ body weight), whereas piglets in the control group received the same volume of sterile saline. Pigs were euthanised $24 \mathrm{~h}$ after the last administration of LPS or saline to obtain intestinal mucosae for biochemical analysis. Compared with the control group, LPS administration decreased $(P<0.05)$ the oxidation of AKG, oleic acid, glutamine and glucose in enterocytes, decreased concentrations of ATP in the duodenal and jejunal mucosae and decreased adenylate energy charge (AMP:ATP ratio) in the jejunal and ileal mucosae. Additionally, LPS treatment reduced $(P<0.05)$ mucosal concentrations of phosphorylated AMPK in the jejunum and ileum as well as acetyl-CoA carboxylase in all segments of the small intestine. The adverse effects of LPS were reversed by AKG. Collectively, these results indicate that dietary supplementation with $1 \%$ AKG beneficially modulates the AMPK signalling pathway to improve energy status in the small intestine of LPS-challenged piglets.

Key words: : $\boldsymbol{\alpha}$-Ketoglutarate: Intestinal mucosae: AMP-activated protein kinase signalling: Piglets: Lipopolysaccharide

$\alpha$-Ketoglutarate (AKG) is an intermediate in the citric acid cycle as well as a precursor of glutamate and glutamine ${ }^{(1)}$, therefore potentially playing an important role in intestinal energy metabolism ${ }^{(2,3)}$. Emerging evidence shows that, similar to glutamine ${ }^{(4)}$, AKG can regulate gene expression and the mammalian target of rapamycin signalling pathway in the pig intestine ${ }^{(5)}$. There is also a suggestion that AKG may have a sparing effect on glutamate and aspartate in cells by serving as a fuel source ${ }^{(6,7)}$, and these amino acids are important for arginine metabolism in young pigs ${ }^{(8)}$. Consistent with these previous reports, we have recently demonstrated that dietary supplementation with $1 \%$ AKG improved small-intestinal histological morphology and absorptive function in weanling piglets challenged with lipopolysaccharide (LPS) ${ }^{(5)}$. However, little is known about the molecular mechanisms responsible for the action of AKG on the small intestine. In the present study, we focused on small-intestinal energy status, because the gut has a high requirement for ATP to support intestinal integrity, function (including nutrient digestion and absorption) and health ${ }^{(1,3)}$

AMP-activated protein kinase (AMPK) is a serine/threonine protein kinase consisting of three subunits $(\alpha, \beta$ and $\gamma)$ and widely exists in eukaryotic cells ${ }^{(9)}$. AMPK is activated by phosphorylation of the $\alpha$-subunit at threonine $172^{(10)}$ and serves as a sensor of cellular energy status ${ }^{(11)}$. AMPK phosphorylates and inactivates one of its downstream target enzymes, acetyl-CoA carboxylase (ACC) ${ }^{(12)}$, which converts acetyl-CoA into malonyl-CoA (an inhibitor of fatty acid oxidation and a substrate for fatty acid synthesis). The present investigation tested the hypothesis that AKG could affect the expression

Abbreviations: ACC, acetyl-COA carboxylase; AEC, adenylate energy charge; AMPK, AMP-activated protein kinase; AKG, $\alpha$-ketoglutarate; LPS, lipopolysaccharide; TBS, Tris-buffered saline; TBS-T, TBS/0.1\% Tween 20.

*Corresponding authors: Dr Y. Hou, fax +86 27 83956175, email houyq777@yahoo.com.cn; Dr G. Wu, email g-wu@tamu.edu 
of total AMPK and ACC proteins, phosphorylated levels of AMPK and ACC, substrate (fatty acid, glucose and glutamine) oxidation, as well as mucosal concentrations of ATP, ADP and AMP. The study presented here is a continuation of our previously published study ${ }^{(5)}$.

\section{Materials and methods}

\section{Experimental design}

The animal protocol for the present study was approved by the Animal Care and Use Committee of Hubei Province. All piglets used in this experiment were born naturally at term (114 $\mathrm{d}$ of gestation). A total of eighteen crossbred (Duroc $\times$ Large White $\times$ Landrace, $7 \cdot 25$ (SD 0.23$) \mathrm{kg}$ ) female pigs weaned at 21 (SD 1) d of age from two litters were assigned by weight and litter to one of three treatment groups: (1) non-challenged (control group, piglets fed the basal diet and receiving an intraperitoneal administration of sterile saline); (2) LPS-challenged control (LPS group, piglets fed the basal diet and receiving an intraperitoneal administration of Escherichia coli $\mathrm{LPS}$ ); (3) LPS $+1 \cdot 0 \% \mathrm{AKG}$ (LPS + AKG group, piglets fed the basal diet supplemented with $1.0 \%$ AKG and receiving an intraperitoneal administration of LPS). After removal from sows, piglets were housed individually in $1.20 \times 1.10 \mathrm{~m}^{2}$ stainless-steel kennels and maintained in an environmentally controlled room $\left(22-25^{\circ} \mathrm{C}\right)^{(5,13)}$. During the experimental period, pigs had free access to drinking-water and a maize- and soyabean meal-based diet $^{(5)}$ to meet the National Research Council's (1998) requirements of nutrients for growing swine (Table 1).

Each of the three treatment groups had six pigs. LPS was dissolved in sterile saline. In the AKG diet, $1.0 \%$ maize starch in the basal diet was replaced by $1.0 \%$ AKG (purity $\geq 99.8 \%$ ), and AKG (powder) was well mixed with the basal diet. All diets were isoenergetic. The dosage of $1 \%$ AKG was chosen, because it could improve intestinal morphology and function in LPS-challenged pigs ${ }^{(5)}$.

The first $3 \mathrm{~d}$ (days $0-3$ post-weaning) was an adjustment period for weanling pigs to adapt to a solid food, and sample collection was performed in the following $16 \mathrm{~d}$. On days 10,12, 14 and 16 of the trial, overnight-fasted piglets in the LPS and LPS + AKG groups were administered intraperitoneally with LPS at $80 \mu \mathrm{g} / \mathrm{kg}$ body weight, whereas pigs in the control group were injected intraperitoneally with the same volume of the vehicle (sterile saline). LPS (E. coli serotype 055:B5; Sigma Chemical, Inc., St Louis, MO, USA) was dissolved in a sterile physiological solution (500 mg LPS/1 saline). During days $0-10$ of the trial (pre-challenge), all pigs were allowed free access to feed and drinking-water. In order to exclude the possible effects of LPS-induced food intake reduction on gastrointestinal characteristics of the pigs, during days 10-16 of the trial (post-challenge), the control and LPS + AKG piglets were fed the same amount of food/kg body weight as LPS piglets. On day 17 (24h after the last LPS challenge or saline administration), all pigs were euthanised under anaesthesia with an intravenous injection of sodium pentobarbital $(50 \mathrm{mg} / \mathrm{kg} \text { body weight })^{(5)}$.
Table 1. Composition and nutrient contents of the basal diet (air-dry basis)

\begin{tabular}{|c|c|}
\hline Items & Content \\
\hline \multicolumn{2}{|l|}{ Ingredients (\%) } \\
\hline Maize & $57 \cdot 88$ \\
\hline Soyabean meal & $20 \cdot 98$ \\
\hline Wheat middlings & 3.00 \\
\hline Fishmeal & $5 \cdot 00$ \\
\hline Dried whey & 3.00 \\
\hline Soya protein concentrate & $2 \cdot 50$ \\
\hline $\mathrm{CaHPO}_{4}$ & $1 \cdot 25$ \\
\hline Premix ${ }^{*}$ & 1.00 \\
\hline Limestone & 0.69 \\
\hline Soya oil & $2 \cdot 50$ \\
\hline Acidifier & 0.30 \\
\hline $\mathrm{NaCl}$ & 0.30 \\
\hline Mould inhibitor & $0 \cdot 10$ \\
\hline Choline chloride & $0 \cdot 20$ \\
\hline L-Lysine. $\mathrm{HCl}(78 \cdot 8 \%$ lysine) & 0.25 \\
\hline DL-Methionine (99\% methionine) & 0.05 \\
\hline Maize starch $†$ & $1 \cdot 00$ \\
\hline \multicolumn{2}{|l|}{ Nutrient composition } \\
\hline Digestible energył (MJ/kg) & $14 \cdot 22$ \\
\hline Crude protein§ (\%) & $20 \cdot 90$ \\
\hline Total lysineł (\%) & $1 \cdot 15$ \\
\hline Total methionine $\ddagger$ (\%) & 0.30 \\
\hline Total methionine + cystine $\neq(\%)$ & 0.65 \\
\hline Total threoninef $(\%)$ & 0.74 \\
\hline Total tryptophan $\ddagger(\%)$ & $0 \cdot 21$ \\
\hline Ca§ (\%) & $0 \cdot 70$ \\
\hline Total P§ (\%) & 0.60 \\
\hline Available P¥ (\%) & 0.32 \\
\hline \multicolumn{2}{|c|}{ 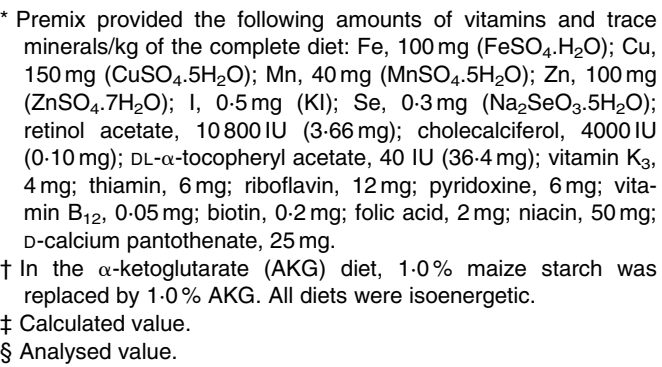 } \\
\hline
\end{tabular}

\section{Intestinal sample collection}

The pig abdomen was opened immediately, and the whole gastrointestinal tract was immediately exposed ${ }^{(5)}$. The small intestine was dissected free of the mesentery and sampled on a chilled stainless-steel tray. The $10 \mathrm{~cm}$ segments were cut at every distal duodenum, mid-jejunum and mid-ileum, respectively. The intestinal segments were opened longitudinally, and the contents were flushed with ice-cold PBS. Mucosal samples were rapidly collected by scraping using a sterile glass microscope slide, rapidly frozen in liquid $\mathrm{N}_{2}$, and then stored at $-80^{\circ} \mathrm{C}$ until analysis. All samples were collected within $10 \mathrm{~min}$ after the pigs were killed.

\section{Determination of substrate oxidation in enterocytes}

Jejunal enterocytes were isolated from the control, LPS-treated and LPS + AKG-treated pigs, using Ca-free Krebs bicarbonate buffer $^{(14)}$. The viability of enterocytes, as assessed by trypan blue exclusion, did not differ among the treatment groups of 
pigs $(92-94 \%)$. Cells were incubated at $37^{\circ} \mathrm{C}$ for $30 \mathrm{~min}$ in a complete oxygenated $\left(95 \% \mathrm{O}_{2} / 5 \% \mathrm{CO}_{2}\right)$ medium ( $\mathrm{pH} 7 \cdot 4$ ) containing $5 \mathrm{~mm}$-D-glucose, $0.5 \mathrm{~mm}$-oleic acid, $2 \mathrm{~mm}$-L-glutamine and other amino acids at physiological levels found in the pig plasma ${ }^{(15)}$. The medium contained D-[U- $\left.{ }^{14} \mathrm{C}\right]$ glucose, $\left[1-{ }^{14} \mathrm{C}\right]$ oleic acid or $\mathrm{L}-\left[\mathrm{U}-{ }^{14} \mathrm{C}\right]$ glutamine. Some media also included $2 \mathrm{~mm}-\mathrm{AKG}+\left[\mathrm{U}_{-}{ }^{14} \mathrm{C}\right] \mathrm{AKG}$. At the end of a $30 \mathrm{~min}$ period of incubation, ${ }^{14} \mathrm{CO}_{2}$ was collected in $0.2 \mathrm{ml}$ of NCS-II ${ }^{(16)}$, and its radioactivity was determined using a Packard liquid scintillation counter ${ }^{(17)}$. The specific radioactivity of D-[U- ${ }^{14}$ C]glucose, $\quad\left[1-{ }^{14} \mathrm{C}\right]$ oleic acid, L-[U- $\left.{ }^{14} \mathrm{C}\right]$ glutamine or $\left[\mathrm{U}_{-}{ }^{14} \mathrm{C}\right] \mathrm{AKG}$ in the incubation medium was used to calculate the rates of $\mathrm{CO}_{2}$ production from D-[U- $\left.{ }^{14} \mathrm{C}\right]$ glucose, $\left[1-{ }^{14} \mathrm{C}\right]$ oleic acid, L-[U- $\left.{ }^{14} \mathrm{C}\right] g l u t a m i n e$ or $\left[\mathrm{U}_{-}{ }^{14} \mathrm{C}\right] \mathrm{AKG}$, respectively ${ }^{(17)}$.

\section{Determination of mucosal ATP, ADP and AMP}

Frozen mucosal samples $(50-100 \mathrm{mg})$ were homogenised with $1 \mathrm{ml}$ pre-cooling $1.5 \mathrm{M}$-perchloric acid in an ice-bath ${ }^{(18)}$. The homogenates were centrifuged at $3000 \boldsymbol{g}$ for $10 \mathrm{~min}$ at $4^{\circ} \mathrm{C}$. An aliquot of the supernatant fluid $(0.5 \mathrm{ml})$ was neutralised with $0.2 \mathrm{ml}$ of $2 \mathrm{M}$-potassium carbonate in ice, and the solution was centrifuged at $3000 \mathrm{~g}$ for $10 \mathrm{~min}$ at $4^{\circ} \mathrm{C}$. The supernatant fluid was stored at $-80^{\circ} \mathrm{C}$ until analysis using HPLC.

The chromatographic system consisted of the Waters Breeze HPLC system (Waters Corporation, Milford, MA, USA), including 1525 binary HPLC pumps, a 2487 Dual- $\lambda$ Absorbance Detector, a 717 plus autosampler and Breeze system software, and a chromatographic column (Waters XBridge C18; $5 \mu \mathrm{m}$, $4.6 \mathrm{~mm} \times 150 \mathrm{~mm})$. The mobile phase $\left(50 \mathrm{~mm}_{-} \mathrm{K}_{2} \mathrm{HPO}_{4}-\right.$ $\mathrm{KH}_{2} \mathrm{PO}_{4}$ buffer solution and methanol (chromatographic grade); 77:23, v/v; $\mathrm{pH} 7 \cdot 0$ ) was filtered through a $0.45 \mu \mathrm{m}$ filter membrane and degassed $15 \mathrm{~min}$ before use. The detection wavelength was $260 \mathrm{~nm}$, the column temperature was $35^{\circ} \mathrm{C}$ and the pump flow rate was $1.0 \mathrm{ml} / \mathrm{min}$. The frozen sample was filtered through a $0.20 \mu \mathrm{m}$ filter membrane after being thawed at room temperature, and the injection volume was $10 \mu \mathrm{l}$. Peaks were identified by their retention times using authentic standards (Sigma Chemical, Inc.). Total adenine nucleotide and adenylate energy charges (AEC) were calculated according to the following equation ${ }^{(19)}$ :

$$
\begin{gathered}
\mathrm{TAN}=\mathrm{ATP}+\mathrm{ADP}+\mathrm{AMP} \\
\mathrm{AEC}=(\mathrm{ATP}+0.5 \mathrm{ADP}) /(\mathrm{ATP}+\mathrm{ADP}+\mathrm{AMP}) .
\end{gathered}
$$

where TAN is the total adenine nucleotide.

\section{Western blot analysis}

Approximately $100 \mathrm{mg}$ of the mucosal samples were quickly placed into the pre-cooling $2 \mathrm{ml}$ centrifuge tubes, mixed with $1 \mathrm{ml}$ of a pre-cooling mixture of radioimmunoprecipitation assay lysis buffer (SC-24948; Santa Cruz Biotechnology, Inc., Santa Cruz, CA, USA) and phosphate inhibitor cocktail (P2850; Sigma Chemical, Inc.), vortexed for $30 \mathrm{~s}$ and homogenised in an ice-bath. The homogenate was centrifuged at $12000 \mathrm{~g}$ for $15 \mathrm{~min}$ at $4^{\circ} \mathrm{C}$. The supernatant fluid was divided into aliquots for Western blot and protein analyses. The concentration of protein was measured using a bicinchoninic acid protein assay kit (Sigma Chemical, Inc.), and then the supernatant fluid was diluted to the same concentrations. For measuring the levels of total and phosphorylated AMPK- $\alpha$, total ACC and phosphorylated ACC- $\beta, 2 \times$ SDS sample buffer ( $2 \mathrm{ml}$ of $0.5 \mathrm{~mm}$-Tris, $\mathrm{pH} 6 \cdot 8,2 \mathrm{ml}$ of glycerol, $2 \mathrm{ml}$ of $10 \%$ SDS, $0 \cdot 2 \mathrm{ml}$ of $\beta$-mercaptoethanol, $0.4 \mathrm{ml}$ of a $4 \%$ solution of bromophenol blue and $1.4 \mathrm{ml}$ of water) was added to the diluted supernatant in a 1:1 ratio, boiled for $5 \mathrm{~min}$ and cooled on ice before use. Mucosal protein $(150 \mu \mathrm{g} / \mathrm{sample}$ for AMPK and $90 \mu \mathrm{g} /$ sample for ACC) was subjected to SDSPAGE using 10\% (for AMPK) or 6\% (for ACC) polyacrylamide resolving gels and transferred to the polyvinylidene difluoride membranes in transfer buffer $(25 \mathrm{~mm}$-Tris base, $0 \cdot 2 \mathrm{M}$-glycine and $20 \%$ methanol, $\mathrm{pH} 8.5$ ). Thereafter, the blotted membranes were washed with $25 \mathrm{ml}$ Tris-buffered saline (TBS), which was prepared in 1 litre of $10 \times$ TBS: $24.2 \mathrm{~g}$ Tris base and $80 \mathrm{~g} \mathrm{NaCl}$ (pH adjusted to $7 \cdot 6$ with $\mathrm{HCl}$ ). The membranes were blocked with $5 \%$ non-fat dry milk in TBS/0.1\% Tween 20 (TBS-T) for $1 \mathrm{~h}$ at room temperature with slow agitation followed by three washes in TBS-T. The membranes were incubated overnight at $4{ }^{\circ} \mathrm{C}$ either with anti-phospho-AMPK $\alpha$ $\left(\mathrm{Thr}^{172}\right.$ ) (no. 2535, rabbit mAb; Cell Signaling, Danvers, MA, USA) and anti-AMPK $\alpha$ (no. 2532; Cell Signaling) or with anti-phospho-ACC- $\beta$ (Ser ${ }^{79}$ ) (no. 3661; Cell Signaling) and anti-ACC (no. 3662; Cell Signaling) with TBS-T containing 5\% bovine serum albumin, at a dilution of 1:1000. The membranes were washed three times with TBS-T and incubated with a secondary antibody (horseradish peroxidase-conjugated goat anti-rabbit IgG, 1:5000; Sigma Chemical, Inc.) at room temperature for $1 \mathrm{~h}$. Blots were developed using an enhanced chemiluminescence substrate (SuperSignal ${ }^{\circledR}$; Pierce Biotechnology, Rockford, IL, USA), visualised using an imaging system (Bio-Rad Versa Doc, Model-5000; Bio-Rad, Hercules, CA, USA) and quantified using Quantity One software.

\section{Statistical analysis}

Values are expressed as means and standard deviations. Experimental data were analysed by one-way ANOVA. Differences among treatment means were determined by Duncan's multiple range test. All statistical analyses were performed using SPSS 13.0 software (SPSS, Inc., Chicago, IL, USA). $P \leq 0.05$ was taken to indicate significance.

\section{Results}

\section{Oxidation of substrates}

Rates of $\mathrm{CO}_{2}$ production from all carbons of AKG, glucose and glutamine as well as from the carbon 1 of oleic acid in pig enterocytes are shown in Table 2. Administration of LPS to pigs decreased $(P<0.05)$ the oxidation of all the four substrates. Dietary supplementation with AKG to the LPS-treated pigs partially ameliorated $(P<0.05)$ the reduction in the oxidation of AKG, glucose, glutamine and oleic acid in enterocytes. 
Table 2. Oxidation of D-glucose, L-glutamine and oleic acid in the jejunal enterocytes ${ }^{\star}$ of lipopolysaccharide (LPS)-challenged weanling pigs receiving dietary supplementation with or without $1 \% \alpha$-ketoglutarate (AKG) (Mean values and standard deviations, $n 6$ )

\begin{tabular}{|c|c|c|c|c|c|c|}
\hline \multirow[b]{2}{*}{ Substrate oxidation } & \multicolumn{2}{|c|}{ Control group $\dagger$} & \multicolumn{2}{|c|}{ LPS group $\ddagger$} & \multicolumn{2}{|c|}{ LPS + AKG group§ } \\
\hline & Mean & SD & Mean & SD & Mean & SD \\
\hline \multicolumn{7}{|c|}{ Production of $\mathrm{CO}_{2}(\mathrm{nmol} / \mathrm{mg}$ protein per $30 \mathrm{~min})$} \\
\hline $2 \mathrm{~mm}-\left[\mathrm{U}-{ }^{14} \mathrm{C}\right] \mathrm{AKG}$ & $53.9^{\mathrm{a}}$ & $4 \cdot 1$ & $32 \cdot 4^{\mathrm{C}}$ & $2 \cdot 6$ & $41 \cdot 7^{\mathrm{b}}$ & 3.0 \\
\hline $5 \mathrm{mM}-\mathrm{D}-\left[\mathrm{U}-{ }^{14} \mathrm{C}\right] \mathrm{Glucose}$ & $32 \cdot 6^{\mathrm{a}}$ & $2 \cdot 7$ & $20 \cdot 1^{\mathrm{c}}$ & $1 \cdot 8$ & $26 \cdot 4^{\mathrm{b}}$ & $2 \cdot 3$ \\
\hline $2 \mathrm{mM}-\mathrm{L}-\left[\mathrm{U}-{ }^{14} \mathrm{C}\right]$ Glutamine & $34 \cdot 9^{a}$ & 2.9 & $21 \cdot 5^{\mathrm{C}}$ & $2 \cdot 2$ & $28 \cdot 0^{\mathrm{b}}$ & 2.5 \\
\hline $0.5 \mathrm{~mm}-\left[1-{ }^{14} \mathrm{C}\right.$ Oleic acid & $0.28^{\mathrm{a}}$ & 0.02 & $0.17^{\mathrm{c}}$ & 0.02 & $0.23^{\mathrm{b}}$ & 0.02 \\
\hline
\end{tabular}

a,b,c Mean values within a row with unlike superscript letters were significantly different $(P<0.05)$.

* Enterocytes were isolated from the jejunum of the control, LPS-treated and LPS + AKG-treated pigs.

† Control (non-challenged control), piglets fed a control diet and injected with sterile physiological saline.

¥ LPS (LPS-challenged control), piglets fed the same control diet and challenged with Escherichia coli LPS.

$\S$ LPS + AKG (LPS + 1.0\% AKG), piglets fed a $1.0 \%$ AKG diet and challenged with LPS.

Concentrations of ATP, ADP and AMP in the smallintestinal mucosa

Data on the concentrations of ATP, ADP and AMP in the intestinal mucosa are summarised in Table 3. Compared with the control group, the concentrations of ATP, ADP and AMP in the duodenal mucosae of LPS piglets were decreased by $44 \%(P<0.05), 53 \%(P<0.05)$ and $42 \%(P<0.05)$, respectively. The LPS treatment also reduced $(P<0.05)$ the concentrations of ATP in the jejunal mucosae by $36 \%$, compared with the control piglets. In comparison with the LPS piglets, AKG supplementation increased $(P<0 \cdot 05)$ the concentrations of ATP in the mucosae of the duodenum and jejunum by 44 and $25 \%$, respectively. The concentrations of ADP, AMP and total adenine nucleotide in the duodenal mucosa of LPS piglets were increased $(P<0.05)$ by 58,18 and $44 \%$, respectively.

Compared with the control group, LPS piglets exhibited a decrease $(P<0.05)$ in AEC in the jejunum and ileum, and an increase $(P<0.05)$ in the ratio of AMP:ATP in the jejunum $(P<0.05)$ and ileum (Table 3). In comparison with the LPS piglets, dietary supplementation with $1 \%$ AKG increased $(P<0.05)$ AEC but decreased $(P<0.05)$ AMP:ATP ratios in the mucosae of the jejunum and ileum (Table 3).

Table 3. Effects of dietary supplementation with $1 \% \alpha$-ketoglutarate (AKG) on adenylate purines in the intestinal mucosa of weaned pigs challenged with lipopolysaccharide (LPS)

(Mean values and standard deviations, $n 6$ )

\begin{tabular}{|c|c|c|c|c|c|c|}
\hline \multirow[b]{2}{*}{ Items } & \multicolumn{2}{|c|}{ Control group* } & \multicolumn{2}{|c|}{ LPS group† } & \multicolumn{2}{|c|}{ LPS + AKG group } \\
\hline & Mean & SD & Mean & SD & Mean & SD \\
\hline \multicolumn{7}{|l|}{ Duodenum } \\
\hline ATP ( $\mu \mathrm{g} / \mathrm{g}$ wet wt) & $77 \cdot 1^{\mathrm{a}}$ & 13.8 & $43 \cdot 5^{c}$ & $8 \cdot 3$ & $62 \cdot 8^{\mathrm{b}}$ & 10.9 \\
\hline ADP ( $\mu \mathrm{g} / \mathrm{g}$ wet $w t)$ & $111 \cdot 2^{\mathrm{a}}$ & 11.6 & $52 \cdot 4^{\mathrm{C}}$ & $12 \cdot 3$ & $82 \cdot 6^{\mathrm{b}}$ & 8.7 \\
\hline AMP $(\mu \mathrm{g} / \mathrm{g}$ wet $w t)$ & $196 \cdot 2^{\mathrm{a}}$ & 31.8 & $113 \cdot 8^{\mathrm{b}}$ & 33.5 & $134.4^{\mathrm{b}}$ & $27 \cdot 4$ \\
\hline TAN§ ( $\mu \mathrm{g} / \mathrm{g}$ wet wt) & $377 \cdot 6^{a}$ & $75 \cdot 8$ & $193 \cdot 8^{\mathrm{c}}$ & 57.9 & $278 \cdot 7^{\mathrm{b}}$ & $73 \cdot 4$ \\
\hline$A E C \|$ & 0.33 & 0.06 & 0.36 & 0.05 & 0.39 & 0.09 \\
\hline AMP:ATP & 2.55 & 0.33 & 2.63 & 0.22 & 1.93 & 0.35 \\
\hline \multicolumn{7}{|l|}{ Jejunum } \\
\hline ATP $(\mu \mathrm{g} / \mathrm{g}$ wet wt) & $51 \cdot 2^{a}$ & 3.5 & $32 \cdot 8^{\mathrm{C}}$ & $2 \cdot 6$ & $40 \cdot 9^{b}$ & $2 \cdot 7$ \\
\hline ADP ( $\mu \mathrm{g} / \mathrm{g}$ wet $w t)$ & $81 \cdot 0$ & 10.5 & $71 \cdot 2$ & $5 \cdot 1$ & 77.4 & $13 \cdot 0$ \\
\hline AMP $(\mu \mathrm{g} / \mathrm{g}$ wet wt) & $118 \cdot 2$ & $28 \cdot 6$ & $115 \cdot 0$ & $14 \cdot 3$ & $109 \cdot 7$ & $24 \cdot 2$ \\
\hline TAN ( $\mu \mathrm{g} / \mathrm{g}$ wet $w t)$ & 256.4 & 35.2 & $219 \cdot 4$ & $17 \cdot 7$ & $232 \cdot 8$ & 23.9 \\
\hline AEC & $0.35^{\mathrm{a}}$ & 0.04 & $0.28^{\mathrm{b}}$ & 0.03 & $0.31^{a, b}$ & 0.04 \\
\hline AMP:ATP & $2 \cdot 42^{b}$ & $0 \cdot 16$ & $3.45^{\mathrm{a}}$ & 0.20 & $2 \cdot 76^{\mathrm{b}}$ & 0.17 \\
\hline \multicolumn{7}{|l|}{ Ileum } \\
\hline ATP ( $\mu \mathrm{g} / \mathrm{g}$ wet wt) & $55 \cdot 0$ & $2 \cdot 4$ & 51.9 & 0.92 & $56 \cdot 8$ & 3.9 \\
\hline ADP ( $\mu \mathrm{g} / \mathrm{g}$ wet $w t)$ & $72 \cdot 2$ & 3.9 & 58.4 & 5.9 & $71 \cdot 2$ & $9 \cdot 3$ \\
\hline AMP $(\mu \mathrm{g} / \mathrm{g}$ wet $w t)$ & 114.5 & $9 \cdot 6$ & $140 \cdot 6$ & $10 \cdot 0$ & 103.9 & $15 \cdot 3$ \\
\hline TAN ( $\mu \mathrm{g} / \mathrm{g}$ wet $w t)$ & $247 \cdot 1$ & 23.4 & $236 \cdot 7$ & $20 \cdot 0$ & 227.4 & 21.4 \\
\hline AEC & $0.37^{\mathrm{a}}$ & 0.02 & $0.33^{\mathrm{b}}$ & 0.01 & $0.35^{\mathrm{a}, \mathrm{b}}$ & 0.04 \\
\hline AMP:ATP & $2 \cdot 08^{b}$ & 0.08 & $2.45^{\mathrm{a}}$ & 0.11 & $1.81^{\mathrm{b}}$ & 0.22 \\
\hline
\end{tabular}

TAN, total adenine nucleotide; AEC, adenylate energy charge.

$\mathrm{a}, \mathrm{b}, \mathrm{c}$ Mean values within a row with unlike superscript letters were significantly different $(P<0.05)$.

* Control (non-challenged control), piglets fed a control diet and injected with sterile physiological saline.

$\dagger$ LPS (LPS-challenged control), piglets fed the same control diet and challenged with Escherichia coli LPS.

$\neq$ LPS + AKG (LPS + 1.0\% AKG), piglets fed a $1.0 \%$ AKG diet and challenged with LPS

$\S \mathrm{TAN}=\mathrm{ATP}+\mathrm{ADP}+\mathrm{AMP}$

$\| A E C=(A T P+0.5 A D P) /(A T P+A D P+A M P)$. 


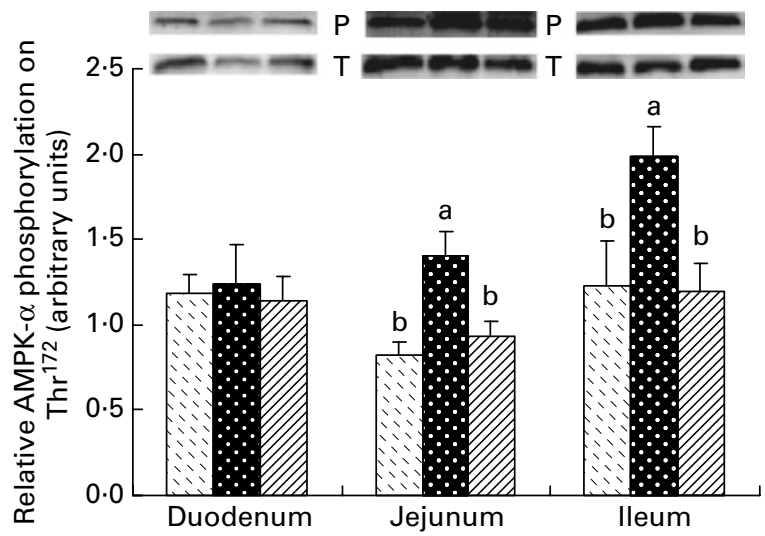

Fig. 1. Phosphorylation state of AMP-activated protein kinase- $\alpha$ (AMPK- $\alpha$ ) in the small-intestinal mucosa of piglets. Mucosal protein extracts were separated by $10 \%$ SDS-PAGE for the determination of the phosphorylation of AMPK- $\alpha(P)$ at threonine 172 and total AMPK- $\alpha(T)$. Values for phosphorylated AMPK- $\alpha$ were normalised for total AMPK- $\alpha$. Values are means, with standard deviations represented by vertical bars $(n 6)$. ๒, Control (nonchallenged control), piglets fed the basal diet and injected with sterile saline; $\mathbf{g}$, lipopolysaccharide (LPS) (LPS-challenged control), piglets fed the basal

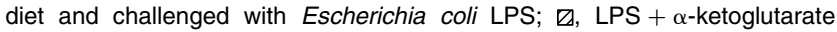
(AKG) (LPS $+1.0 \%$ AKG), piglets fed the basal diet supplemented with $1.0 \%$ AKG and challenged with LPS. ${ }^{a, b}$ Mean values within the same intestinal segment with unlike letters were significantly different $(P<0.05)$.

\section{Phosphorylated levels for AMP-activated protein kinase} and acetyl-CoA carboxylase

Phosphorylation at $\mathrm{Thr}^{72}$ for the $\alpha$-isoform of AMPK is illustrated in Fig. 1. LPS challenge resulted in an increase $(P<0.05)$ in the ratio of phosphorylated AMPK- $\alpha$ :AMPK- $\alpha$ in the jejunal and ileal mucosae, compared with the control piglets. Relative to LPS piglets receiving no LPS, dietary supplementation with $1 \%$ AKG decreased $(P<0.05)$ the phosphorylated AMPK- $\alpha$ :AMPK in the jejunum and ileum to the value observed for the control group. However, in the duodenum, the levels of phosphorylated AMPK- $\alpha$ did not differ $(P>0.05)$ among the control, LPS and LPS + AKG piglets.

Phosphorylation at $\mathrm{Ser}^{79}$ for ACC- $\beta$ and total ACC- $\beta$ protein is illustrated in Fig. 2. Compared with the control group, the ratio of phosphorylated ACC- $\beta$ :ACC- $\beta$ in the duodenal, jejunal and ileal mucosae of LPS piglets was increased $(P<0.05)$ by 38, 49 and $40 \%$, respectively. Compared with the LPS group, dietary supplementation with AKG did not affect $(P>0.05)$ the phosphorylated ACC- $\beta: A C C-\beta$ in the small-intestinal mucosa of LPS piglets.

\section{Discussion}

There is growing interest in the nutritional regulation of mucosal inflammation in both clinical medicine and animal production, because the gastrointestinal tract is the first defence against diet-derived pathogens ${ }^{(20)}$. AKG displays remarkable metabolic and regulatory versatility in the pig intestine ${ }^{(5,7)}$, but little is known about its significance in gut signalling or function. The results of the present study indicate, for the first time to our knowledge, that AKG beneficially prevented
LPS-induced alterations in substrate oxidation (Table 2), cellular energy status (Table 3) and protein levels for phosphorylated AMPK and ACC- $\beta$ (Fig. 1) in the piglet small intestine.

LPS is a molecule found in the membrane of all gramnegative bacteria and can induce alterations in gastrointestinal oxygen metabolism ${ }^{(21)}$. In addition, LPS increased heat shock protein 70 expression in the pig small intestine ${ }^{(5)}$, which is an indicative of oxidative stress in intestinal cells ${ }^{(22)}$. Moreover, LPS can cause mitochondrial injury in the small intestine ${ }^{(23)}$. Extending these previous reports, we observed that LPS treatment reduced ATP concentrations and AEC, while altering the cellular energy status in piglet intestinal mucosae (Table 3 ). This is in keeping with our new findings that the intestinal oxidation of glucose, glutamine and fatty acids was reduced in response to LPS administration (Table 2). Thus, the LPSchallenged weanling piglets provide a useful animal model to develop nutritional interventions for improving energy status in the gut.

The energy charge of the adenyl pool is a better measure of the energy state of a tissue than the level of a single nucleotide ${ }^{(19,24)}$. ATP hydrolysis can increase the cellular concentration of ADP, which is converted by adenylate kinase (2 ADP $\leftrightarrow \mathrm{ATP}+\mathrm{AMP})$ to ATP and $\mathrm{AMP}^{(11)}$. Another novel and important finding of the present study is that AKG supplementation resulted in (1) increased ATP, (2) reduced AMP:ATP and (3) increased AEC in the pig small intestine (Table 3). These three lines of evidence indicate that dietary supplementation with AKG could modulate the adenine nucleotide pool and support the notion that AKG beneficially alleviates the LPS-induced damage of intestinal energy metabolism. The results reveal a hitherto unrecognised role for AKG in improving mitochondrial function in the small intestine.

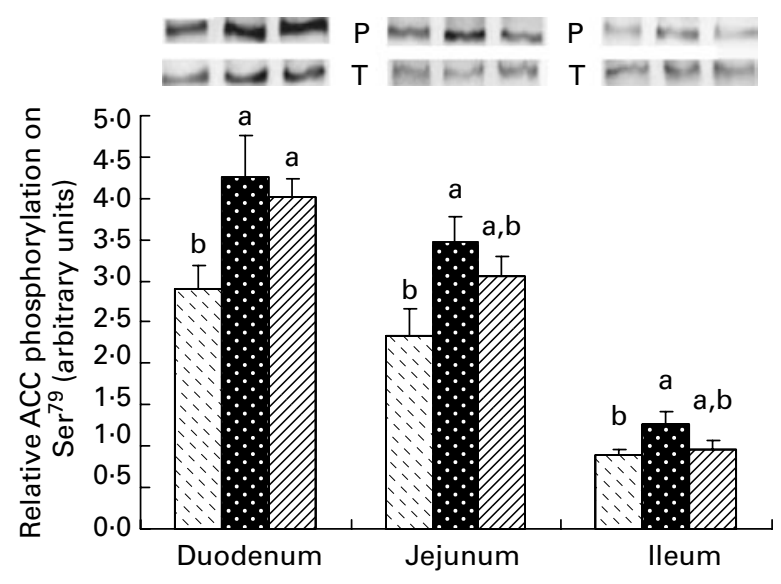

Fig. 2. Phosphorylation state of acetyl-CoA carboxylase (ACC) in the smallintestinal mucosa of piglets. Mucosal protein extracts were separated by $6 \%$ SDS-PAGE for the determination of the phosphorylation of ACC- $\beta(P)$ at $\mathrm{Ser}^{79}$ and total ACC $(\mathrm{T})$. Values for phosphorylated ACC were normalised for total ACC. Values are means, with standard deviations represented by vertical bars $(n 6)$. $⿴$, Control (non-challenged control), piglets fed the basal diet and injected with sterile saline; $\mathbf{s}$, lipopolysaccharide (LPS) (LPS-challenged control), piglets fed the basal diet and challenged with Escherichia coli LPS; $\square$, LPS $+\alpha$-ketoglutarate (AKG) (LPS $+1.0 \%$ AKG), piglets fed the basal diet supplemented with $1.0 \%$ AKG and challenged with LPS. ${ }^{\mathrm{a}, \mathrm{b}}$ Mean values within the same intestinal segment with unlike letters were significantly different $(P<0.05)$. 
Lambert et al. ${ }^{(25)}$ reported that $80 \%$ of dietary AKG was oxidised by the small intestine in young pigs, and the remaining AKG was utilised by other splanchnic tissues. Thus, dietary supplementation with AKG did not increase its concentrations in the porcine portal blood ${ }^{(25)}$. Because AKG is extensively metabolised in the first-pass by the intestinal mucosa and virtually does not enter the portal circulation ${ }^{(25)}$, we suggest that AKG exerts its nutritional benefits in weanling piglets primarily at the small-intestinal level. The possibility that AKG may also affect other splanchnic tissues cannot be ruled out.

AMPK activity in mammalian cells can be regulated by stimuli that affect cellular ATP levels ${ }^{(11)}$. For example, hypoxia, which occurs in the small intestine of LPS-treated animals ${ }^{(21)}$, leads to the activation of AMPK via an increase in the AMP:ATP ratio ${ }^{(26)}$. When activated, AMPK switches on catabolic pathways for ATP regeneration, such as the oxidation of glucose, amino acids and fatty acids, while switching off ATP-requiring pathways, such as fatty acid and TAG synthesis ${ }^{(27)}$. To our knowledge, this is the first study indicating that AKG may regulate AMPK signalling in the intestinal mucosa. At present, it is not known whether AKG directly or indirectly phosphorylates AMPK. AKG may act as an energy substrate or a signalling molecule, or both. It is also possible that the metabolism of AKG in enterocytes may regulate its intracellular concentration and thus its effects on cellular targets (e.g. AMPK). Either the elucidation of these mechanisms for the actions of AKG on the gut via AMPK activation and energy metabolism or the determination of whether ATP depletion in enterocytes may induce the activation of AMPK would require complex biochemical studies, and such studies are beyond the scope of the present study. However, emerging evidence shows that AMPK is an upstream molecule in the mammalian target of rapamycin signalling pathway ${ }^{(28)}$. Thus, we speculate that possibly through the mammalian target of rapamycin activation ${ }^{(5)}$, AKG stimulates AMPK phosphorylation and oxidation of energy substrates in the intestinal mucosa (Table 2), thereby enhancing ATP supply and supporting cell function. Further studies are warranted to test this hypothesis.

Phosphorylated AMPK- $\alpha$ (active) AMPK could inhibit ACC activity in the skeletal muscle and liver by phosphorylating ACC- $\beta$ (namely increasing the phosphorylated level of ACC $)^{(29)}$, but a role for ACC in enterocyte metabolism is unknown. Interestingly, there have been reports suggesting that, in colonic epithelial cells (colonocytes), conversion of acetyl-CoA to malonyl-CoA may play an important role in lipogenesis $^{(30,31)}$. ACC activation is a rate-controlling step in the conversion of acetyl-CoA to malonyl-CoA ${ }^{(32)}$. Malonyl-CoA inhibits carnitine palmitoyl-CoA transferase-1, which transports long-chain fatty acyl-CoA from the cytoplasm into the mitochondria for oxidation ${ }^{(30)}$. Thus, a fall in malonyl-CoA concentration in response to the increased levels of phosphorylated AMPK and phosphorylated ACC may lead to increased oxidation of fatty acids. However, we found that LPS treatment reduced the oxidation of oleic acid in enterocytes (Table 2). This result may be explained by mitochondrial injury or dysfunction in the small intestine of endotoxin-challenged pigs, thereby impairing Krebs cycle activity and the mitochondrial electron transport system. Consistent with our finding is the report that LPS treatment reduced the activity of aconitase in rat myocardial tissue ${ }^{(33)}$. Aconitase is a target of $\mathrm{NO}^{(34)}$, and increased $\mathrm{NO}$ production by inducible $\mathrm{NO}$ synthase in response to LPS treatment could be a mechanism responsible for the reduced oxidation of substrates in the enterocytes of LPS-challenged pigs. Notably, oxidation of oleic acid in the enterocytes of LPS-treated pigs was increased by AKG supplementation, indicating the improvement of mitochondrial integrity and function.

In summary, dietary supplementation with AKG beneficially improves the energy status of the intestinal mucosa as well as AMPK activation and ACC inactivation in the intestinal mucosa of LPS-challenged pigs. These novel findings have important implications for the development of new interventions to ameliorate gut injury and dysfunction in animals. Further research is warranted to understand how AKG affects the AMPK signalling in the gut.

\section{Acknowledgements}

The present study was jointly supported by the National Natural Science Foundation of China (no. 30871801, 30901041, 30901040, 30928018), the Program for Innovative Research Groups of Hubei Provincial Natural Science Foundation (no. 2007ABC009), National Research Initiative Competitive Grants from the Animal Growth and Nutrient Utilization Program (no. 2008-35206-18764) of the USDA National Institute of Food and Agriculture, Texas AgriLife Research (H-8200), and the Thousand-People Talent program at China Agricultural University. Y. H. and G. W. designed the study and wrote the manuscript. K. Y., L. W., B. D., D. F., Y. L., H. Z., J. L. and Y. Li collected and analysed the experimental results. P. K. and Y. Y. participated in the experiments and revised the manuscript. All authors contributed to data interpretation and approved the final version of the manuscript. There is no conflict of interest that should be disclosed.

\section{References}

1. Blachier F, Boutry C, Bos C, et al. (2009) Metabolism and functions of L-glutamate in the epithelial cells of the small and large intestines. Am J Clin Nutr 90, 814S-821S.

2. Baker DH (2009) Advances in protein-amino acid nutrition of poultry. Amino Acids 37, 29-41.

3. Burrin DG \& Stoll B (2009) Metabolic fate and function of dietary glutamate in the gut. Am J Clin Nutr 90, 850S-856S.

4. Rhoads JM \& Wu G (2009) Glutamine, arginine, and leucine signaling in the intestine. Amino Acids 37, 111-122.

5. Hou YQ, Wang L, Ding BY, et al. (2010) Dietary $\alpha$-ketoglutarate supplementation ameliorates intestinal injury in lipopolysaccharide-challenged piglets. Amino acids 39, 555-564.

6. Junghans P, Derno M, Pierzynowski S, et al. (2006) Intraduodenal infusion of $\alpha$-ketoglutarate decreases whole body energy expenditure in growing pigs. Clin Nutr 25, 489-496.

7. Lambert BD, Filip R, Stoll B, et al. (2006) First-pass metabolism limits the intestinal absorption of enteral $\alpha$-ketoglutarate in young pigs. $J$ Nutr 136, 2779-2784. 
8. Wu G, Bazer FW, Davis TA, et al. (2007) Important roles for the arginine family of amino acids in swine nutrition and production. Livest Sci 112, 8-22.

9. Stapleton D, Mitchelhill KI, Gao G, et al. (1996) Mammalian AMP-activated protein kinase subfamily. J Biol Chem 271, 611-614.

10. Aymerich I, Foufelle F, Ferré P, et al. (2006) Extracellular adenosine activates AMP-dependent protein kinase (AMPK). J Cell Sci 119, 1612-1621.

11. Hardie DG (2003) Minireview: the AMP-activated protein kinase cascade: the key sensor of cellular energy status. Endocrinology 144, 5179-5183.

12. Dyck JR, Kudo N, Barr AJ, et al. (1999) Phosphorylation control of cardiac acetyl-CoA carboxylase by cAMP-dependent protein kinase and 5'-AMP activated protein kinase. Eur J Biochem 262, 184-190.

13. He QH, Kong XF, Wu G, et al. (2009) Metabolomic analysis of the response of growing pigs to dietary L-arginine supplementation. Amino Acids 37, 199-208.

14. Wu G, Knabe DA, Yan W, et al. (1995) Glutamine and glucose metabolism in enterocytes of the neonatal pig. $\mathrm{Am}$ J Physiol Regul Integr Comp Physiol 268, R334-R342.

15. Wu G, Davis PK, Flynn NE, et al. (1997) Endogenous synthesis of arginine plays an important role in maintaining arginine homeostasis in postweaning growing pigs. $J$ Nutr 127, 2342-2349.

16. Li XL, Bazer FW, Gao H, et al. (2009) Amino acids and gaseous signaling. Amino Acids 37, 65-78.

17. Chen LX, Li P, Wang JJ, et al. (2009) Catabolism of nutritionally essential amino acids in developing porcine enterocytes. Amino Acids 37, 143-152.

18. Haynes TE, Li P, Li XL, et al. (2009) L-Glutamine or L-alanyl-Lglutamine prevents oxidant- or endotoxin-induced death of neonatal enterocytes. Amino Acids 37, 131-142.

19. Atkinson DE (1968) The energy charge of the adenylate pool as a regulatory parameter. Interaction with feedback modifiers. Biochemistry 7, 4030-4034.

20. Wu G (2009) Amino acids: metabolism, functions, and nutrition. Amino Acids 37, 1-17.

21. Menguy R (1981) Role of gastric mucosal energy metabolism in the etiology of stress ulceration. World J Surg 5, 175-180.

22. Sepponen K \& Poso AR (2006) The inducible form of heat shock protein 70 in the serum, colon and small intestine of the pig: comparison to conventional stress markers. Vet J 171, 519-524.

23. Lobo SM, Backer DD, Sun QH, et al. (2003) Gut mucosal damage during endotoxic shock is due to mechanisms other than gut ischemia. J Appl Physiol 95, 2047-2054.

24. McKnight JR, Satterfield MC, Jobgen WS, et al. (2010) Beneficial effects of L-arginine on reducing obesity: potential mechanisms and important implications for human health. Amino Acids 39, 349-357.

25. Lambert BD, Stoll B, Niinikoski H, et al. (2002) Net portal absorption of enterally fed $\alpha$-ketoglutarate is limited in young pigs. J Nutr 132, 3383-3386.

26. Evans AM, Mustard KJW, Wyatt CN, et al. (2005) Does AMPactivate protein kinase couple inhibition of mitochondrial oxidative phosphorylation by hypoxia to calcium signaling in $\mathrm{O}_{2}$-sensing cells? J Biol Chem 280, 41504-41511.

27. Winder WW \& Hardie DG (1999) AMP-activated protein kinase, a metabolic master switch: possible roles in type 2 diabetes. Am J Physiol Endocrinol Metab 277, E1-E10.

28. Hong-Brown LQ, Brown CR, Kazi AA, et al. (2010) Alcohol and PRAS40 knockdown decrease mTOR activity and protein synthesis via AMPK signaling and changes in mTORC1 interaction. J Cell Biochem 109, 1172-1184.

29. Carling D (2004) The AMP-activated protein kinase cascade - a unifying system for energy control. Trends Biochem Sci 29, $18-24$.

30. Andriamihaja M, Chaumontet C, Tome D, et al. (2009) Butyrate metabolism in human colon carcinoma cells: implications concerning its growth inhibitory effect. J Cell Physiol 218, 58-65.

31. Zambell KL, Fitch MD \& Fleming SE (2003) Acetate and butyrate are the major substrates for de novo lipogenesis in rat colonic epithelial cells. J Nutr 133, 3509-3515.

32. Jobgen WS, Fried SK, Fu WJ, et al. (2006) Regulatory role for the arginine-nitric oxide pathway in metabolism of energy substrates. J Nutr Biochem 17, 571-588.

33. Mason KE \& Stofan DA (2008) Endotoxin challenge reduces aconitase activity in myocardial tissue. Arch Biochem Biophys 469, 151-156.

34. Wu G, Bazer FW, Davis TA, et al. (2009) Arginine metabolism and nutrition in growth, health and disease. Amino Acids 37, $153-168$. 